\title{
Uma discussão sobre oscilações quirais e inversão de spin
}

(A discussion about chiral oscillations and spin flipping)

\author{
Alex Eduardo de Bernardini ${ }^{1}$ e Stefano De Leo ${ }^{2}$ \\ ${ }^{1}$ Instituto de Física Gleb Wataghin, Universidade Estadual de Campinas, Campinas, SP, Brasil \\ ${ }^{2}$ Departamento de Matemática Aplicada, Universidade Estadual de Campinas, Campinas, SP, Brasil \\ Recebido em 4/2/2005; Revisado em 28/9/2005; Aceito em 18/10/2005
}

\begin{abstract}
Neste trabalho desenvolvemos uma breve discussão sobre o conceito de quiralidade que pode aparecer ao construirmos uma lagrangiana de interação da teoria eletrofraca. Para melhor contextualizarmos os conceitos que estamos estudando, introduzimos uma breve descrição do status atual do problema de oscilação quântica de neutrinos. De fato, temos na quiralidade, assim como na helicidade, um caráter quântico de importância fundamental no estudo de física de neutrinos e de física de partículas de maneira geral. Neste contexto, os efeitos da oscilação quiral podem ser explicados como uma implicação do fenômeno de zitterbewegung que emerge quando soluções da equação de Dirac são usadas para descrever a evolução espaço-temporal de um pacote de ondas. Procuramos demonstrar, no entanto, que não existe vínculo entre tal fenômeno e o processo de inversão de spin, o qual vem associado ao número quântico que determina a helicidade de uma partícula que se propaga no espaço-tempo.
\end{abstract}

Palavras-chave: quiralidade, helicidade, oscilações.

In this paper we present a brief discussion on the concept of chirality which can appear when we construct the interacting lagrangian of the electroweak theory. In order to put the concepts we are studying in a clearer context, we introduce a brief description of the status of neutrino quantum oscillation problem. In fact, the chirality as well as the helicity correspond to a quantum character of fundamental importance in the study of neutrino physics or, generically, of particle physics. In this context, the effects of chiral oscillation can be explained as a consequence of the zitterbewegung phenomenon which emerges when Dirac equation solutions are used for describing the space-time evolution of a wave packet. We demonstrate, however, that there is not a constraint between such a phenomenon and the spin flipping process which is related to the quantum number of helicity of the propagating particle.

Keywords: chirality, helicity, oscillations.

\section{Introdução}

Recentemente, um grande interesse no fenômeno quântico de oscilação $[1,2]$ tem estimulado de maneira crescente a produção de trabalhos, em particular, no contexto da física de neutrinos [3, 4], onde, sob o ponto de vista teórico, procura-se descrever a mistura quântica de sabores $[5,6]$ e os mecanismos teóricos de oscilações quânticas de sabor [2, 7]. Apesar de bastante explorado e muito discutido nos últimos anos, as dificuldades conceituais que aparecem na interpretação do fenômeno de oscilação quântica, assim como outras características e fenômenos físicos envolvendo as partículas oscilantes, representam, ainda, um intrigante, e as vezes embaraçante, desafio para os físicos.

De fato, nos últimos três anos, experimentos de estudo do fluxo natural de neutrinos têm providenciado as mais fortes evidências de neutrinos massivos e da

\footnotetext{
${ }^{1}$ E-mail: alexeb@ifi.unicamp.br.

Copyright by the Sociedade Brasileira de Física. Printed in Brazil.

superposição de estados quânticos do mesmo. Destacamos os resultados do experimento Super-Kamiokande com neutrinos atmosféricos [8], no qual uma significante assimetria up-down em eventos com múons de altas energias é observada. Temos também os resultados do experimento SNO com neutrinos solares [9] no qual foram obtidas evidências diretas da transição de neutrinos eletrônicos (solares) em neutrinos de outros sabores. Temos ainda os resultados do experimento KamLAND [10] que confirmam o desaparecimento de neutrinos eletrônicos principalmente devido ao mecanismo de oscilação de sabores. Além do grande interesse em descrever o mecanismo correto para a conversão de sabores, a dificuldade conceitual no entendimento de alguns aspectos físicos que se "escondem" nas fórmulas de oscilação ainda representam um extenso campo para o desenvolvimento de estudos teóricos.

Ainda com relação aos dados experimentais, todos 
os fenômenos observados experimentalmente poderiam ser explicados caso assumíssemos a existência de apenas três números quânticos de sabor para os neutrinos: eletrônico, muônico e tauônico, não fosse a anomalia observada no experimento LSND [11, 12, 13] que permite a interpretação da existência de um quarto neutrino o qual deveria ser inerte (não interagente). $\mathrm{Na}$ verdade, conforme discutido em [6], ocorre que, no experimento LSND é feita a medida de um fluxo de neutrinos produzidos pelo decaimento de um píon em "voô" $\left(\pi^{+} \rightarrow \mu^{+} \nu_{\mu}\right)$ e pelo decaimento de um antimúon em repouso $\left(\mu^{+} \rightarrow e^{+} \nu_{e} \bar{\nu}_{\mu}\right)$ de modo que se observa, no ponto de detecção, um déficit de antineutrinos eletrônicos. Quando o mecanismo standard de oscilação quântica de sabores [14] é levado em consideração, os dados referentes ao déficit observado apontariam para uma diferença quadrática de massas $\Delta m_{L S N D}^{2} \approx 1 \mathrm{eV}^{2}$ muito maior do que o valor $\Delta m^{2} \approx 10^{-3} \mathrm{eV}^{2}$ obtido a partir de outros experimentos. O fato é que com os dados obtidos no LSND [12], o esquema de mistura quântica com três sabores de neutrinos acaba por não ser satisfatório. Seria necessária (no mínimo) uma outra "espécie" de neutrino para permitir o valor tão elevado de $\Delta m_{L S N D}$. Com três sabores de neutrinos é possível definir apenas dois valores independentes para as diferenças quadráticas de massas, as quais já foram determinadas por meio dos dados sobre neutrinos solares, atmosféricos, de reatores e de aceleradores até o momento obtidos [6]. Uma sugestão para a explicação desta anomalia vinda dos dados experimentais do LSND seria a da suposição da existência de um quarto neutrino, caracterizado como um singleto do Modelo Padrão que, entretanto, por meio de algum mecanismo (de oscilação), interagisse com os demais neutrinos ativos. Contudo, alguns experimentos (KARMEN 2 [15] e MiniBoone [16]) que podem ou poderiam confirmar os dados vindos do LSND, ainda não foram finalizados de modo fornecer dados conclusivos sobre $\Delta m_{L S N D}$ e sobre o número de sabores, ou mais diretamente, sobre o número de neutrinos. Apesar da ausência de confirmação experimental, que atuaria como um aspecto de motivação adicional no desenvolver de nossos cálculos teóricos, assumiremos que as transições de estados mistos, onde o estado final de um neutrino corresponde a uma mistura quântica de uma componente ativa e uma componente estéril, são ainda aceitáveis.

De fato, a hipótese de mistura entre as já conhecidas espécies de neutrinos (eletrônico, muônico e tauônico) e neutrinos com uma massa mais elevada não é recente na literatura científica $[17,18]$. Ao mesmo tempo, a inversão de spin atribuída a alguns mecanismos dinâmicos de interação externa $[19,20]$ chegou a ser englobado no estudo do déficit observacional envolvendo neutrinos solares. Em particular, como conseqüência de um momento magnético não nulo que interage com um campo eletromagnético externo, neutrinos com helicidade lefthanded teriam o número quântico correspondente modificado (para right-handed). Os efeitos sobre oscilações de sabor devidos a interações com campos magnéticos externos, em um tipo de fenômeno onde a quiralidade tenha sido preservada, também já foram introduzidos na literatura [21], porém, carecem de um estudo teórico pormenorizado. Observamos, contudo, que somente para neutrinos em regimes ultra-relativísiticos de propagação a mudança de helicidade seria equivalente à mudança de quiralidade. Os neutrinos com quiralidade positiva (right) não se acoplam às correntes de carga fraca que "absorvem" 2 neutrinos. Conseqüentemente, essas partículas se tornam estéreis com respeito às interações fracas. Independentemente da existência de campos externos, como os neutrinos são detectados essencialmente via correntes de carga fraca do tipo VA (interação vetorial menos pseudo-vetorial (axial)), a oscilação quiral poderia, conforme se observa pela construção do mecanismo teórico de oscilação de quiralidade, ocupar um espaço significativo no problema de oscilação quântica de sabores envolvendo neutrinos [22], contudo, como veremos, não é o que acontece sob as atuais circunstâncias experimentais.

Dado o interesse científico crescente, e na procura de permitir ao leitor uma maior familiaridade com os conceitos de quiralidade e helicidade, dentre as discussões propostas neste manuscrito, descreveremos o mecanismo de oscilação quiral para partículas fermiônicas soluções da equação de Dirac. Neste modelo, os neutrinos permanecem como autoestados de massa, porém, contêm uma componente de quiralidade (positiva) estéril. Os efeitos de oscilação podem ser explicados como uma implicação da interferência entre componentes de freqüência positiva e negativa soluções da equação de Dirac em um fenômeno semelhante ao zitterbewegung $[23,24]$ da posição, que emerge quando soluções da equação de Dirac são usadas para descrever a evolução espaço-temporal de um pacote de ondas. Salientamos ainda que várias contribuições científicas $[22,25]$ têm surgido no âmbito de descrever o mecanismo de oscilação quiral [26] acoplado ao fenômeno de oscilação quântica de sabores com pacotes de ondas de Dirac [22].

Como proposta introdutória, na seção 2, em busca de sanar uma pequena confusão na conceituação de quiralidade e de helicidade que freqüentemente se observa na literatura, apenas ampliamos um pouco a discussão do caráter quiral das interações eletrofracas, descrevemos as analogias no confronto entre os conceitos de quiralidade e helicidade e, por fim, recapitulamos de forma bastante simplificada o quadro geral de modelos de gauge com simetria left-right [27]. Na seção 3, após introduzirmos o processo de oscilação quiral, discutimos o caráter físico da escolha, e mesmo da construção, do estado inicial envolvido no fenômeno de oscilação. Para

\footnotetext{
${ }^{2}$ Como em um vértice de interação fraca.
} 
concretizarmos nosso estudo, na seção 4, analisamos o fenômeno de inversão de spin (spin-flipping) em comparação com processo de oscilação quiral. A seção 5 se presta às considerações finais.

\section{A equação de Dirac e o conceito de transformação quiral}

A equação de Dirac para uma partícula livre em sua notação covariante é dada por

$$
\left(i \gamma^{\mu} \partial_{\mu}-m\right) \psi(x)=0
$$

ou, na forma conjugada, por

$$
i \partial_{\mu} \bar{\psi}(x) \gamma^{\mu}+m \bar{\psi}(x)=0,
$$

onde sempre utilizaremos a notação padronizada convenientemente adotada na literatura [23, 24, 28]. Podemos estabelecer as soluções de ondas planas para a equação de Dirac que descreve uma partícula livre $\operatorname{como} \psi(x)=\psi_{+}(x)+\psi_{-}(x) \operatorname{com}$

$$
\begin{array}{ll}
\psi_{+}(x)=\exp [-i p x] u(p) & \text { para freqüências positivas e } \\
\psi_{-}(x)=\exp [+i p x] v(p) & \text { para freqüências negativas, }
\end{array}
$$

onde $p$ é o quadrimomento da partícula spinorial, $p=(E, \mathbf{p})$ com $E^{2}=m^{2}+\mathbf{p}^{2}$, tal qual possamos escrever:

$$
\left(\gamma^{\mu} p_{\mu}-m\right) u(p)=\left(\gamma^{\mu} p_{\mu}+m\right) v(p)=0 .
$$

A álgebra das matrizes de Dirac $\left(\gamma^{\mu}\right)$ possibilita que reescrevamos a Eq. (4) no referencial de repouso de modo a obter [23]

$$
\left(\gamma^{0}-m\right) u(m, 0)=\left(\gamma^{0}+m\right) v(m, 0)=0,
$$

que implica em:

$$
u^{1,2}(m, 0)=\begin{gathered}
\xi^{1,2} \\
0
\end{gathered} \quad \text { e } \quad v^{1,2}(m, 0)=\begin{gathered}
0 \\
\eta^{1,2}
\end{gathered},
$$

onde $\eta^{1,2}=\xi^{1,2}=\left(\begin{array}{l}1 \\ 0\end{array}\right),\left(\begin{array}{l}0 \\ 1\end{array}\right)$. Com os resultados de (5) podemos efetuar um boost de Lorentz de modo a obter as seguintes expressões gerais [23]:

$$
\begin{aligned}
& u^{s}(p)=\frac{\gamma^{\mu} p_{\mu}+m}{[2 E(m+E)]^{\frac{1}{2}}} u^{s}(m, 0)=@_{0}^{0} \frac{\frac{E+m}{\boldsymbol{\sigma}_{\cdot \mathbf{p}}^{2}}}{[2 E(E+m)]^{\frac{1}{2}}} \xi^{\frac{1}{2}} \xi^{s}{ }_{1}^{\mathrm{A}}, \\
& v^{s}(p)=\frac{-\gamma^{\mu} p_{\mu}+m}{[2 E(m+E)]^{\frac{1}{2}}} v^{s}(m, 0)=@ \frac{\boldsymbol{\sigma} \cdot \mathbf{p}}{[2 E(E+m)]^{\frac{1}{2}}} \eta^{s} \mathrm{~A}
\end{aligned}
$$

e da mesma maneira, $\bar{u}(x)$ (ou $\bar{v}(x)$ ) definido conforme a aplicação de $\bar{u}(x)=u^{\dagger}(x) \gamma^{0}\left(\right.$ ou $\left.\bar{v}(x)=v^{\dagger}(x) \gamma^{0}\right)$.

Para uniformizarmos a notação na discussão das propriedades quirais dos spinores de Dirac, utilizaremos a representação de Dirac das matrizes gamma $\left(\gamma^{\mu}\right)$ descritas por:

$$
\begin{aligned}
& \gamma^{0}=\begin{array}{rr}
\mathbf{1} & 0 \\
0 & -\mathbf{1}
\end{array} \quad, \quad \gamma^{i}=\begin{array}{ccc}
0 & \sigma^{i} \\
-\sigma^{i} & 0
\end{array} \quad \mathrm{e} \\
& \gamma^{5}=\begin{array}{ll}
0 & \mathbf{1} \\
\mathbf{1} & 0
\end{array} \text {. }
\end{aligned}
$$

de modo que as soluções da equação de Dirac sejam dadas pela expressão (7). A matriz $\gamma^{5}$ de Dirac tem as seguintes propriedades:

$$
\gamma^{5 \dagger}=\gamma^{5}, \quad \gamma^{52}=1 \quad \text { e } \quad \gamma^{5}, \gamma^{\mu}=0,
$$

sendo que a última delas implica na relação de comutação $\left[\gamma^{5}, S^{\mu \nu}\right]=0$, onde $S^{\mu \nu}=\frac{i}{2}\left[\gamma^{\mu}, \gamma^{\nu}\right]$ são os geradores das transformações contínuas de Lorentz. Para estendermos o estudo das propriedades que envolvem a matriz $\gamma^{5}$, consideraremos as solução geral da equação de Dirac obtida, por meros motivos de simplificação matemática, ao assumirmos uma análise em uma dimensão espacial $z$, descrita por:

$$
\begin{aligned}
& \psi(\mathbf{x}, t)=^{\mathrm{Z}} \frac{d p^{3}}{(2 \pi)^{3}}{ }_{s=1,2}^{\mathrm{X}}\left\{b^{s}(p) u^{s}(p) \exp [-i p x]+\right. \\
& \left.d^{s *}(p) v^{s}(p) \exp [+i p x]\right\},
\end{aligned}
$$

de modo que podemos definir as densidades de corrente (de simetria) vetoriais e pseudo-vetoriais dadas respectivamente por:

$$
\begin{aligned}
j^{\mu}(\mathbf{x}, t) & =\bar{\psi}(\mathbf{x}, t) \gamma^{\mu} \psi(\mathbf{x}, t) \mathrm{e} \\
j^{\mu 5}(\mathbf{x}, t) & =\bar{\psi}(\mathbf{x}, t) \gamma^{\mu} \gamma^{5} \psi(\mathbf{x}, t) .
\end{aligned}
$$

Se assumimos que $\psi(\mathbf{x}, t)$ satisfaz a equação de Dirac, podemos calcular o divergente dessas densidades de corrente,

$$
\begin{aligned}
\partial_{\mu} j^{\mu}(\mathbf{x}, t) & =\left(\partial_{\mu} \bar{\psi}(\mathbf{x}, t)\right) \gamma^{\mu}\left(\partial_{\mu} \psi(\mathbf{x}, t)\right) \\
& =(i m \bar{\psi}(\mathbf{x}, t)) \psi+\bar{\psi}(-i m \psi(\mathbf{x}, t))=0
\end{aligned}
$$

que implica na imediata conservação de $j^{\mu}(\mathbf{x}, t)$. Ao acoplarmos um campo $\psi(\mathbf{x}, t)$ de Dirac a um campo eletromagnético, $j_{\mu}(\mathbf{x}, t)$ passa a descrever uma densidade de corrente elétrica.

De maneira similar, podemos calcular:

$$
\partial_{\mu} j^{\mu 5}(\mathbf{x}, t)=2 i m \bar{\psi}(\mathbf{x}, t) \gamma^{5} \psi(\mathbf{x}, t) .
$$

Se $m=0$, esta uma, também chamada de densidade de corrente axial, também é conservada. Em geral, é comum definir os termos de densidades de corrente left$L$ e right- $R$ por meio de combinações lineares com o operador $\gamma^{5}$,

$$
\begin{aligned}
j_{L}^{\mu}(\mathbf{x}, t) & =\bar{\psi}(\mathbf{x}, t) \gamma^{\mu} \frac{1-\gamma^{5}}{2} \psi(\mathbf{x}, t) \quad \mathrm{e} \\
j_{R}^{\mu}(\mathbf{x}, t) & =\bar{\psi}(\mathbf{x}, t) \gamma^{\mu} \frac{1+\gamma^{5}}{2} \psi(\mathbf{x}, t) .
\end{aligned}
$$

Somente se $m=0$, estas seriam densidades de corrente de partículas com caráter de helicidade left-handed e right-handed e seriam separadamente conservadas. As duas correntes $j^{\mu}(\mathbf{x}, t)$ e $j^{\mu 5}(\mathbf{x}, t)$ são as correntes de 
Noether que correspondem às duas transformações de gauge representadas respectivamente por

$$
\psi(\mathbf{x}, t) \rightarrow \exp [i \alpha] \psi(\mathbf{x}, t)
$$

e

$$
\psi(\mathbf{x}, t) \rightarrow \exp \left[i \alpha \gamma^{5}\right] \psi(\mathbf{x}, t) .
$$

A primeira delas descreve uma simetria global da Lagrangiana de Dirac. A segunda, chamada de transformação quiral, representa uma simetria apenas da parte cinética da Lagrangiana de Dirac. O teorema de Noether ratifica que uma densidade de corrente axial relacionada a uma transformação quiral se conserva somente se $m=0$ [29].

\subsection{Helicidade e quiralidade}

Vejamos, agora, o que acontece quando escolhemos convenientemente os spinores de duas componentes $\eta^{s}$ e $\xi^{s}$ como autoestados da matriz de Pauli $\sigma^{z}$, ou seja, como na $\mathrm{Eq} \cdot(6)$,

$$
\eta^{1,2}=\xi^{1,2}=\begin{array}{ll}
1 \\
0
\end{array} \quad, \quad \begin{aligned}
& 0 \\
& 1
\end{aligned}
$$

Ao escolhermos o eixo- $z$ como o eixo diretor do vetor momento $\mathbf{p}$, tal que $p_{x}=p_{y}=0$, podemos definir um operador $h$ que, para esta escolha específica, será dado por:

$$
h=\boldsymbol{\Sigma} \cdot \hat{\boldsymbol{p}}=\frac{1}{2} \boldsymbol{\sigma} \cdot \hat{\boldsymbol{p}} \quad \begin{array}{ll}
1 & 0 \\
0 & 1
\end{array}=\frac{1}{2} \quad \begin{array}{lll}
\sigma^{z} & 0 \\
0 & \sigma^{z}
\end{array} .
$$

Observamos, então, que $u^{s}\left(p_{z}, m\right)$ e $v^{s}\left(p_{z}, m\right)$ com $s=$ 1,2 são autovetores de $h$ com autovalores $h=+\frac{1}{2}$ para $s=1$ e $h=-\frac{1}{2}$ para $s=2$. Em geral, apesar de soluções de ondas planas para partículas livres poderem sempre ser escolhidas como as autofunções de $h$, não é possível escolher uma solução tal que a mesma seja uma autofunção de $\boldsymbol{\Sigma} \cdot \hat{\boldsymbol{n}}$ com um vetor diretor unitário $\hat{\boldsymbol{n}}$ arbitrário. Isto ocorre porque o operador $\boldsymbol{\Sigma} \cdot \hat{\boldsymbol{n}}$ não comuta com o Hamiltoniano de uma partícula livre, a menos que $\hat{\boldsymbol{n}}= \pm \hat{\boldsymbol{p}}$ ou $\boldsymbol{p}=0$.

O operador $h$ que pode ser diagonalizado simultaneamente com o Hamiltoniano de uma partícula livre é chamado de operador de helicidade [24]. Este operador corresponde exatamente à projeção do spin da partícula em questão na direção do vetor momento $\boldsymbol{p}$. Uma partícula com $h=+\frac{1}{2}$ é chamada de right-handed, enquanto uma outra com $h=-\frac{1}{2}$ é chamada de lefthanded. A helicidade de uma partícula com massa nãonula depende do sistema espaço-temporal de referência, ou seja, a qualquer momento, um boost pode ser feito para um outro sistema de referência onde o momento da partícula pode ser obtido com o sentido oposto àquele inicial. Com o spin inalterado, a helicidade da partícula muda. Para partículas sem massa, contudo, as quais viajam com velocidade da luz, tal boost não é possível.

De certa maneira, helicidade e quiralidade, como números quânticos, carregam informações complementares. A quiralidade é invariante diante de uma transformação de Lorentz contínua, porém, não é constante no tempo se a partícula é maciça. A helicidade de uma partícula livre, por outro lado, é uma constante no tempo mas não corresponde a um invariante de Lorentz. De fato, um autoestado quiral pode sempre ser escrito como uma combinação linear de dois autoestados de helicidade.

\subsection{O conceito de calibre acoplado à quirali- dade}

Um importante aspecto da física de partículas está na possibilidade de acomodarmos o espectro de partículas conhecidas em representações irredutíveis de um determinado grupo de simetria interna. Um tipo importante e particular de simetria interna é a chamada simetria de gauge, utilizada como um princípio dinâmico na construção de teorias de campos. Ao longo do tempo, a idéia de que as interações fundamentais da natureza são determinadas pela simetria de gauge adquiriu uma importância essencial. As teorias dotadas com simetrias de gauge têm como importante propriedade o fato das interações dos campos de gauge serem ditadas pela simetria em questão, reduzindo consideravelmente as possíveis formas de interação. Concomitantemente, as simetrias de gauge introduzem um certo grau de unificação entre as diferentes interações conhecidas, desde que elas passem a ter uma estrutura geométrica comum.

Para observarmos como o conceito de quiralidade se apresenta em uma teoria de gauge, propomos a construção de uma Lagrangiana invariante de gauge em uma teoria, inicialmente, com férmions sem massa. Podemos acoplar um campo de Dirac a um campo de gauge atribuindo uma representação de um grupo de $G$ aos campos quirais- $L, \psi_{i_{L}}(\mathbf{x}, t)$, com quiralidade negativa, e uma outra representação aos campos quirais- $R$, $\psi_{i_{R}}(\mathbf{x}, t)$, com quiralidade positiva. Com isto, podemos escrever

$$
\mathcal{L}=\bar{\psi}(\mathbf{x}, t) \quad \partial_{\mu}-i g A_{\mu}^{a} T^{a} \quad \frac{1-\gamma^{5}}{2} \quad \psi(\mathbf{x}, t),
$$

onde observamos que somente os campos quirais- $L$ se acoplam com os campos $A_{\mu}^{a}$ de bósons de gauge.

Em um passo imediato, é possível verificar que a Lagrangiana (19) é um objeto invariante diante de uma transformação local de gauge [29],

$$
\begin{aligned}
\psi(\mathbf{x}, t) & \rightarrow 1+i \alpha_{\mu}^{a} T^{a} \frac{1-\gamma^{5}}{2} \psi(\mathbf{x}, t), \\
A_{\mu}^{a} & \rightarrow A_{\mu}^{a}+g^{-1} \partial_{\mu} \alpha^{a}+f^{a b c} A^{b} \alpha^{c},
\end{aligned}
$$

onde as propriedades da álgebra em questão são descritas pelas relações de comutação entre os operadores $T^{a}$ dadas por:

$$
\stackrel{\mathrm{h}}{T^{a}, T^{b}} \stackrel{\text { i }}{=} i f^{a b c} T^{c} .
$$

Dado que os campos quirais- $R$ são campos livres, podemos eliminar estes campos de uma vez por todas e escrever a Lagrangiana invariante de gauge para 
férmions puramente quirais- $L$. A idéia de campos de gauge que se acoplam com férmions quirais- $L$ tem um papel central na construção de uma teoria para interações fracas.

Para exibirmos as propriedades dos férmions que se acoplam por meio de interações com quiralidade definida, é conveniente reescrevermos a Lagrangiana com simetria diante da operação de conjugação de carga $C=i \gamma^{2} \gamma^{0}$. Um campo de carga conjugada seria descrito por $\psi^{c}$ como

$$
\psi^{c}=C \bar{\psi}^{T}=i \gamma^{2} \psi^{*},
$$

de modo que o conjugado de uma componente quiral$R$ de um campo $\psi$, sob as transformações de Lorentz, se transforma como uma quantidade quiral- $L$ que seria definida por $\psi_{L}^{c}$, isto é,

$$
\begin{aligned}
\psi_{L}^{c}= & \left(\psi^{c}\right)_{L}=i \frac{1-\gamma^{5}}{2} \gamma^{2} \gamma^{0} \gamma^{0} \psi^{*}= \\
& i \gamma^{2} \frac{1+\gamma^{5}}{2} \quad \psi^{*}=i \gamma^{2} \gamma^{0} \psi_{R}^{*} \equiv\left(\psi_{R}\right)^{c},
\end{aligned}
$$

e, da mesma maneira, o conjugado de componente quiral- $L$ de um campo $\psi$ se transforma como uma quantidade quiral- $R$ que seria definida por $\psi_{R}^{c}$

$$
\psi_{R}^{c}=i \gamma^{2} \gamma^{0} \psi_{L}^{*} \equiv\left(\psi_{L}\right)^{c} .
$$

Tal propriedade é importante para observarmos o que ocorre em um modelo de calibre com simetria $L$ $R$. Primeiramente, é possível definir os multipletos que caracterizam as partículas envolvidas nas interações com os bósons de gauge e suas propriedades de transformação descritas pelos geradores do grupo que rege as interações características do modelo. Dentre tentativas consolidadas, ou ainda promissoras, temos o modelo $S U(2)_{L} \otimes S U(2)_{R} \otimes U(1)_{B-l}$ de Mohapatra e Pati [27] e os modelos com simetria $S U(3) \otimes S U(4)_{L}\left(S U(3)_{L}\right) \otimes$ $U(1)$ de Pleitez e outros [30,31], além de uma série de extensões [32, 33] onde sucessivas quebras espontâneas de simetria (QES) resultam na "recuperação" do modelo GWS [34] para interações eletrofracas [35, 36]. Em todos esses modelos, os neutrinos de caráter quiral- $R$ aparecem como partículas interagentes em multipletos que se acoplam com bósons de gauge descritos pelos geradores dos grupos em questão (antes da QES). Entretanto, pela própria característica vetorial das interações mediadas pelos bósons de calibre, a possibilidade de conversão de quiralidade $L-R$ mediante um acoplamento de calibre é impossível uma vez que o acoplamento vetorial entre campos de caracteres quirais opostos tem amplitude de probabilidade nula ${ }^{3}$,

$$
\begin{aligned}
\bar{\psi}_{L} A_{\mu} \gamma^{\mu} \psi_{L} & \equiv \overline{\psi_{R}} A_{\mu} \gamma^{\mu} \psi_{L} \\
& \propto\left(1+\gamma^{5}\right) \psi^{\dagger} \gamma^{0} A_{\mu} \gamma^{\mu}\left(1-\gamma^{5}\right) \psi \\
& =\psi^{\dagger}\left(1+\gamma^{5}\right) \gamma^{0} A_{\mu} \gamma^{\mu}\left(1-\gamma^{5}\right) \psi \\
& =\psi^{\dagger} \gamma^{0} A_{\mu} \gamma^{\mu}\left(1+\gamma^{5}\right)\left(1-\gamma^{5}\right) \psi=0 .
\end{aligned}
$$

Dada esta impossibilidade, na próxima seção descrevemos a maneira através da qual oscilações quirais podem ser efetivamente descritas.

\section{Oscilações quirais}

Se uma partícula sem massa representada por um spinor de Dirac é produzida em um autoestado quiral (do operador $\gamma^{5}$ ), então, a sua quiralidade será uma constante do movimento, além de representar uma quantidade invariante de Lorentz. Esta propriedade não se mantém, de modo algum, para partículas massiças. Para elucidarmos este ponto temos o Hamiltoniano para partícula livre dado por

$$
H_{0}=-i \gamma^{0} \gamma \cdot \partial+m \gamma^{0},
$$

o qual representa o operador de evolução temporal para tempos subseqüentes ao tempo $(t=0)$ de criação de um spinor, e não comuta com o operador quiral $\gamma^{5}$ devido ao termo de massa em $H_{0}$. De modo a sermos mais precisos, observamos que

$$
-i \partial_{t}\left\langle\gamma^{5}\right\rangle=\left\langle\left[H_{0}, \gamma^{5}\right]\right\rangle=2 m\left\langle\gamma^{0} \gamma^{5}\right\rangle
$$

onde $m$ é a massa relativa a um spinor o qual assumimos que seja um autoestado de massa. Ao admitirmos que simplificações matemáticas substanciais aparecem quando adotamos uma análise em uma dimensão espacial $z$, o valor médio do lado direito da equação acima é dado explicitamente por:

$$
\left\langle\gamma^{0} \gamma^{5}\right\rangle={ }_{-\infty}^{\mathrm{Z}+\infty} d z \psi^{\dagger}(z, t) \gamma^{0} \gamma^{5} \psi(z, t),
$$

onde $\psi(z, t)$ pode ser descrita em termos de soluções de ondas planas pela expansão:

$$
\begin{aligned}
\psi(z, t)= & \quad{\frac{d p_{z}}{2 \pi}}_{s=1,2}^{\mathrm{Z}+\infty}\left\{b^{s}\left(p_{z}, m_{1}\right) u^{s}\left(p_{z}, m_{1}\right) \exp \left[-i\left(E\left(p_{z}, m_{1}\right) t-p_{z} z\right)\right]\right. \\
& \left.+d^{s *}\left(p_{z}, m_{1}\right) v^{s}\left(p_{z}, m_{1}\right) \exp \left[+i\left(E\left(p_{z}, m_{1}\right) t-p_{z} z\right)\right]\right\}
\end{aligned}
$$

\footnotetext{
${ }^{3}$ Observe que segundo a notação adotada aqui, a qual é diferente de alguns livros textos, $\bar{\psi}_{R} \psi_{L}=\bar{\psi}_{L} \psi_{L}=0$ onde $\bar{\psi}_{R(L)}$ é um objeto de quiralidade- $R(L)$.
} 
que satisfaz a equação de Dirac. Entretanto, da mesma maneira que para o efeito de zitterbewegung [23, 24], a fórmula (27) não implica em um efeito físico necessariamente observável. É fácil observar que, para um pacote de ondas construído somente com soluções de ondas planas com freqüências positivas, a equação de Dirac para spinores $u\left(p_{z}, m\right)$ e $v\left(p_{z}, m\right)$ leva a um valor médio nulo para a expressão (27),

$$
\begin{aligned}
& \left(\gamma^{0} E\left(p_{z}, m\right)-\gamma^{3} p_{z}-m\right) u\left(p_{z}, m\right)= \\
& \bar{u}\left(p_{z}, m\right)\left(\gamma^{0} E\left(p_{z}, m\right)-\gamma^{3} p_{z}-m\right)=0, \\
& \left(\gamma^{0} E\left(p_{z}, m\right)-\gamma^{3} p_{z}+m\right) v\left(p_{z}, m\right)= \\
& \bar{v}\left(p_{z}, m\right)\left(\gamma^{0} E\left(p_{z}, m\right)-\gamma^{3} p_{z}+m\right)=0 .
\end{aligned}
$$

Para ilustrarmos este ponto, calculamos explicitamente:

$$
\begin{aligned}
\left\langle\gamma^{0} \gamma^{5}\right\rangle= & { }_{-\infty}^{\mathrm{Z}+\infty} d z \sum_{-\infty}^{\mathrm{Z}+\infty} \frac{d k_{z}}{2 \pi}{ }_{-\infty}^{\mathrm{Z}+\infty}{\frac{d p_{z}}{2 \pi}}_{s, s^{\prime}}^{\mathrm{X}} b^{s *}\left(p_{z}, m\right) b^{s^{\prime}}\left(k_{z}, m\right) \bar{u}^{s}\left(p_{z}, m\right) \gamma^{5} u^{s^{\prime}}\left(k_{z}, m\right) \\
\times & \times \exp \left[i\left(E\left(p_{z}, m\right)-E\left(k_{z}, m\right)\right) t\right] \exp \left[-i\left(p_{z}-k_{z}\right) z\right] \\
= & { }_{-\infty}^{+\infty}{\frac{d p_{z}}{2 \pi}}_{s, s^{\prime}}^{\mathrm{X}} b^{s *}\left(p_{z}, m\right) b^{s^{\prime} *}\left(p_{z}, m\right) \bar{u}^{s}\left(p_{z}, m\right) \gamma^{5} u^{s^{\prime}}\left(p_{z}, m\right),
\end{aligned}
$$

assim, como conseqüência da identidade (unidimensional) de Gordon "quiral", temos:

$$
\begin{aligned}
& u^{s \dagger}\left(k_{z}, m\right) \gamma^{0} \gamma^{5} u^{s^{\prime}}\left(p_{z}, m\right)= \\
& \frac{1}{2 m} u^{s \dagger}\left(k_{z}, m\right) \gamma^{0} \quad E\left(k_{z}, m\right) \gamma^{0} \gamma^{5}-k_{z} \gamma^{3} \gamma^{5} \\
& +\gamma^{5} \gamma^{0} E\left(p_{z}, m\right)-\gamma^{5} \gamma^{3} p_{z} \quad u^{s^{\prime}}\left(p_{z}, m\right) .
\end{aligned}
$$

de onde observamos que:

$$
u^{s \dagger}\left(p_{z}, m\right) \gamma^{0} \gamma^{5} u^{s^{\prime}}\left(p_{z}, m\right)=0,
$$

o qual leva a um valor nulo para a média calculada de $\gamma^{0} \gamma^{5}$. Uma prova similar é obtida quando trabalhamos com soluções de freqüência negativa.

Por outro lado, ao trabalharmos simultaneamente com freqüências positivas e negativas, o termo de interferência entre soluções de freqüências com sinais opostos leva a uma componente de oscilação quiral.

$$
\begin{aligned}
\left\langle\gamma^{0} \gamma^{5}\right\rangle= & { }_{-\infty}^{\mathrm{Z}+\infty} d z{ }_{-\infty}^{\mathrm{Z}+\infty} \frac{d k_{z}}{2 \pi}{ }_{-\infty}^{\mathrm{Z}+\infty}{\frac{d p_{z}}{2 \pi}}_{s, s^{\prime}}^{\mathrm{X}} d^{s}\left(k_{z}, m\right) b^{s^{\prime}}\left(p_{z}, m\right) \bar{v}^{s}\left(k_{z}, m\right) \gamma^{5} u^{s^{\prime}}\left(p_{z}, m\right) \\
& \left.\times \exp \left[-i\left(E\left(p_{z}, m\right)+E\left(k_{z}, m\right)\right) t\right] \exp \left[i\left(p_{z}+k_{z}\right) z\right]-\text { h.c. }\right\} \\
= & \mathrm{Z}+\infty{\frac{d p_{z}}{2 \pi}}_{s, s^{\prime}}^{\mathrm{X}} \mathrm{n} d^{s}\left(-p_{z}, m\right) b^{s^{\prime}}\left(p_{z}, m\right) \bar{v}^{s}\left(-p_{z}, m\right) \gamma^{5} u^{s^{\prime}}\left(p_{z}, m\right) \exp \left[-2 i E\left(p_{z}, m\right) t\right]-\text { h.c. } .
\end{aligned}
$$

A identidade de Gordon "quiral", para termos cruzados (interferência), levaria a:

$$
\begin{aligned}
& v^{s \dagger}\left(k_{z}, m\right) \gamma^{0} \gamma^{5} u^{s^{\prime}}\left(p_{z}, m\right)= \\
& -\frac{1}{2 m} v^{s \dagger}\left(k_{z}, m\right) \gamma^{0} \quad E\left(k_{z}, m\right) \gamma^{0} \gamma^{5}-k_{z} \gamma^{3} \gamma^{5} \\
& -\gamma^{5} \gamma^{0} E\left(p_{z}, m\right)-\gamma^{5} \gamma^{3} p_{z} \quad u^{s^{\prime}}\left(p_{z}, m\right),
\end{aligned}
$$

que proporciona o resultado:

$$
\partial_{t}\left\langle\gamma^{5}\right\rangle=\underbrace{\mathrm{Z}+\infty}_{-\infty} \frac{d p_{z}}{2 \pi}{ }_{s}^{\mathrm{X}}-(2 i m) d^{s}\left(-p_{z}, m\right) b^{s^{\prime}}\left(p_{z}, m\right) \exp \left[-2 i E\left(p_{z}, m\right) t\right]+\text { h.c. },
$$

de onde, após perfazermos a integral temporal, obtemos:

$$
\left\langle\gamma^{5}\right\rangle(t)=\left\langle\gamma^{5}\right\rangle(0)+{ }_{-\infty}^{\mathrm{Z}+\infty} \frac{d p_{z}}{2 \pi} \frac{m}{E\left(p_{z}, m\right)} \times{ }_{s}^{\mathrm{X}} d^{s}\left(-p_{z}, m\right) b^{s^{\prime}}\left(p_{z}, m\right)\left\{\exp \left[-2 i E\left(p_{z}, m\right) t\right]-1\right\}+\text { h.c. } .
$$


De uma maneira muito particular, De Leo e Rotelli [26] discutem o real significado físico da oscilação quiral calculada a partir dos resultados apresentados até aqui. Demonstra-se que, para uma função de onda representada inicialmente por uma gaussiana, a qual tem um valor médio de quiralidade nulo, as oscilações de quiralidade $L \leftrightarrow R$ (left $\leftrightarrow$ right) se cancelam de modo que não se observa o caráter oscilante de maneira efetiva. Em princípio, isto poderia originar um aparente paradoxo. De fato, este é o ponto inicial de uma discussão da influência do caráter quiral do estado inicial no fenômeno de oscilação quântica.

\subsection{O estado inicial em um processo de os- cilação quiral}

Para reproduzirmos as idéias contidas em [26], consideramos que para qualquer autoestado de massa representado por uma onda plana solução da equação de Dirac, a função de onda no sistema de repouso representa sempre uma mistura igual de componentes de quiralidade $L-R$. Isto é facilmente verificado quando:

$$
\psi=\frac{1-\gamma^{5}}{2} \psi+\frac{1+\gamma^{5}}{2} \psi=\psi_{L}+\psi_{R},
$$

onde $\psi_{L, R}$ correspondem respectivamente à quiralidade ғ1. Pode ser demonstrado que, no sistema de repouso de uma partícula, teríamos:

$$
\left|\psi_{L}\right|^{2}=\left|\psi_{R}\right|^{2} .
$$

Note que este resultado não é invariante diante de uma transformação de Lorentz pois esta não é unitária. Desta maneira, enquanto temos uma seção de choque invariante de Lorentz, temos, também, probabilidades quirais que não são invariantes. Isto vem a sugerir que as medidas de probabilidade não dependem da quiralidade do objeto oscilante, ou seja, temos aparentemente um argumento contrário à relevância física que as oscilações quirais poderiam apresentar.

A resposta a esta objeção construída com base na invariância de Lorentz da seção de choque é que, para qualquer sistema de referência, as oscilações quirais são efetivamente importantes devido a projeção na forma "vetorial menos axial" (V-A) das correntes de interação fraca. Mais especificamente, as variações de probabilidades quirais produzidas por estas transformações de Lorentz (mesmo se $\gamma^{5}$ comuta com os geradores de Lorentz) são automaticamente compensadas pelas condições de normalização da função de onda e da forma transformada (de Lorentz) dos bósons vetoriais intermediários e outras partículas que compõem a determinação da seção de choque em questão.

Esta situação é semelhante à aparente contradição entre os seguintes fatos. Em todos os processos (conhecidos) através dos quais neutrinos são produzidos como autoestados quase puros de helicidade lefthanded, dado que são criados dominantemente com estados ultra-relativísticos (onde quiralidade é quase equivalente à helicidade) por meio de uma interação $\mathrm{V}$ A, o valor médio da quiralidade é muito próximo de -1 (ou $L$ ). Mas no sistema de repouso do neutrino (onda plana) este valor médio seria nulo. Visto do referencial (de repouso) do neutrino, não há preferência entre os valores de quiralidade -1 ou +1 . Não há, então, uma contradição com o fato de haver uma dominância de neutrinos com quiralidade- $L$ na natureza? Não. Enquanto existem sistemas de referência comuns a quase todos os neutrinos que se apresentam como autoestados quase puros de quiralidade- $L$, existem muitos outros sistemas de referência, não coincidentes aos anteriores, para os quais a quiralidade- $R$ é dominante para cada neutrino.

Uma outra questão é pertinente: em qual sistema de referência a forma da localização do pacote de ondas deve ser levada em conta. Um pacote de ondas gaussiano, sugerido principalmente pela simplicidade de suas características de integração, certamente não independe do sistema de referência considerado. Desta maneira, se escolhemos, por exemplo, o Sol como um sistema de referência, poderemos estabelecer que os neutrinos são criados como autoestados quase puros de quiralidade- $L$. Isto abre espaço para oscilações quirais que, possivelmente, como veremos na próxima seção, poderiam implicar em uma diminuição do fluxo efetivo, isto é, detectados mediante interação fraca, dos neutrinos que chegam até a Terra.

\section{Analogias com processos de inversão de spin}

A interação de partículas fermiônicas representadas por $\psi(\mathbf{x}, t)$ em coordenadas espaciais tridimensionais com campos vetoriais externos pode ser representada por um termo de Lagrangiana de interação descrito por:

$$
-G \bar{\psi}_{\alpha}(\mathbf{x}, t) \gamma_{\mu} V^{\mu}(\mathbf{x}) \psi_{\alpha}(\mathbf{x}, t),
$$

onde $G$ representa a constante de acoplamento da interação correspondente. No caso de interações fracas cujos férmions envolvidos são neutrinos que se acoplam a um campo bosônico vetorial $Z^{\mu}(\mathbf{x})$, a expressão (42), onde esquecemos por um momento as oscilações de sabores, seria corretamente descrita por:

$$
-\frac{\sqrt{g^{2}+g^{\prime 2}}}{2} \bar{\nu}_{\alpha}(\mathbf{x}, t) \gamma_{\mu} Z^{\mu}(\mathbf{x}) \nu_{\alpha}(\mathbf{x}, t)
$$

onde $g$ é a constante de acoplamento relativa a simetria $S U(2)_{L}$ e $g^{\prime}$ é a constante de acoplamento relativa a simetria $U(1)_{Y}$ da teoria GWS $\left(S U(2)_{L} \times U(1)_{Y}\right)$ [34]. Com o termo de interação adicionado à Lagrangiana de uma partícula livre, o operador Hamiltoniano seria escrito como:

$$
H=-i \gamma_{0} \gamma_{1} \partial^{1}-\frac{\sqrt{g^{2}+g^{\prime 2}}}{2} \gamma_{0} \gamma_{\mu} Z^{\mu}(\mathbf{x})+\gamma_{0} m .
$$

Neste caso, a operação de comutação com o operador de quiralidade $\gamma_{5}$ resulta novamente, como na expressão 
(27), em um termo não nulo advindo somente do termo de massa $\gamma_{0} m$,

$$
\left[\gamma_{5}, H\right]=\left[\gamma_{5}, H_{0}\right]=2 m\left\langle\gamma_{0} \gamma_{5}\right\rangle,
$$

onde, neste caso, porém, o valor médio $\left\langle\gamma_{0} \gamma_{5}\right\rangle$ deve se alterar devido à nova forma assumida pelas novas soluções da equação de Dirac com os termos de interação que aparecem. Contudo, o motivo pelo qual introduzimos esta seção, não é o de prosseguirmos com estes cálculos, mas sim, de observarmos que a presença de um novo termo de interação no Hamiltoniano (44) determina o caráter da evolução temporal do operador helicidade descrito na expressão (18) do início deste capítulo como a projeção do operador de spin na direção e sentido de propagação da partícula fermiônica,

$$
h=\frac{1}{2} \quad \begin{array}{lll}
\boldsymbol{\sigma} \cdot \hat{\boldsymbol{p}} & 0 \\
& 0 & \boldsymbol{\sigma} \cdot \hat{\boldsymbol{p}}
\end{array} .
$$

Em outras palavras, ao interagir com um campo vetorial externo, uma partícula fermiônica deve estar sujeita ao fenômeno de rotação de spin (spin flipping) onde seu estado de polarização se altera. De modo a esclarecermos esta suposição, consideramos o Hamiltoniano no espaço de momentos para uma partícula que se propaga na direção $z$,

$$
H\left(\mathbf{x}, p_{z}\right)=-i \gamma_{0} \gamma_{3} \partial_{z}-\frac{\sqrt{g^{2}+g^{\prime 2}}}{2} \gamma_{0} \gamma_{\mu} Z^{\mu}(\mathbf{x})+\gamma_{0} m
$$

e indicamos a dependência temporal de $h$ como

$$
\begin{aligned}
& -i \partial_{t}\langle h\rangle=\langle[H, h]\rangle= \\
& -\frac{\sqrt{g^{2}+g^{\prime 2}}}{2}\left\langle\gamma_{5} \gamma_{3}\left(\gamma_{1} Z_{1}(\mathbf{x})+\gamma_{2} Z_{2}(\mathbf{x})\right)\right\rangle .
\end{aligned}
$$

Também é importante notar que $\left[\gamma_{5}, h\right]=0$, isto é, a medida do comportamento temporal de uma dessas variáveis não influencia no valor da outra. De maneira mais objetiva, para uma Hamiltoniana descrita como (47), a oscilação de quiralidade jamais podem alterar o estado de polarização de uma partícula fermiônica.

Apesar de, envolvendo algumas manipulações matemáticas um pouco mais extensas, ser possível determinar a evolução temporal do operador $h$ da mesma maneira como fizemos para $\gamma_{5}$, a helicidade não é um invariante de Lorentz ${ }^{4}$. Além disso, interações fracas têm como característica a quiralidade -1 definida, sendo que o mesmo não é válido para a helicidade. A importância do estudo envolvendo estas variáveis, sob o ponto de vista fenomenológico, está relacionada ao fato de que efeitos dinâmicos como oscilação de quiralidade e de helicidade (rotação de spin) alteram, respectivamente, cada qual a sua maneira, a probabilidade de conversão de sabores e os estados de polarização de um neutrino.

\section{Conclusões}

Neste texto confrontamos os conceitos de helicidade e quiralidade e, ao concentrarmos a discussão neste último, observamos que o uso da equação de Dirac como a equação de movimento para partículas fermiônicas descritas por pacotes de ondas que representam os autoestados de massa que produzem o fenômeno de interferência durante a propagação espaço-temporal levam a uma fórmula de oscilação do caráter quiral, isto é, a aparição de férmions com caráter quiral- $R$ que resultam da conversão $L \rightarrow R$ de um estado inicial $L$. É de se salientar que o processo de oscilação quiral pode introduzir pequenas interferências em outros processos físicos, notoriamente, o processo de oscilação quântica de sabores para partículas fermiônicas que se propagam no vácuo em um processo totalmente desacoplado do processo de inversão de spin. Entretanto, com as massas que os neutrinos parecem ter, que para os três diferentes sabores são compatíveis com aproximadamente $0 \mathrm{eV}, 0.002 \mathrm{eV}$ e $0.1 \mathrm{eV}$ fica muito difícil observarmos as implicações efetivas deste mecanismo em regimes (ultra)relativísiticos de propagação [37]. Observamos que o processo de oscilação quiral tem características dinâmicas semelhantes ao de um processo de rotação de spin em um caso restrito de interação com o meio externo, porém, resultam em efeitos físicos completamente diversos: a rotação de spin não influencia, por exemplo, no resultado dos processos de conversão de sabores, enquanto que oscilações quirais jamais alteram o estado de polarização de um neutrino devido a comutabilidade dos operadores de quiralidade e de helicidade. Deste modo, para encerrarmos de maneira conclusiva, esperamos ter contribuído no entendimento de um aspecto pelo qual muitas vezes, na literatura, não é dada a devida atenção: a identificação clara dos conceitos de quiralidade e helicidade no contexto da física de partículas, e a descrição dos fenômenos físicos que daí se derivam.

\section{Agradecimentos}

Este trabalho foi possível graças ao Suporte Financeiro da Coordenação e Aperfeiçoamento de Pessoal de Nível Superior (CAPES) e do Fundo de Apoio à Pesquisa do Estado de São Paulo (FAPESP).

\section{Referências}

[1] M. Zralek, Acta Phys. Polon. B29, 3925 (1998).

[2] M. Beuthe, Phys. Rep. 375, 105 (2003).

[3] K. Zuber, Phys. Rep. 305, 295 (1998).

[4] K. Scholberg, hep-ex/0308011.

[5] M.C. Gonzalez-Garcia and Y. Nir, Rev. Mod. Phys. 75, 245 (2003).

[6] A. de Gouvêa, hep-ph/0503086.

\footnotetext{
${ }^{4} \mathrm{Um}$ boost de Lorentz pode levar um estado de helicidade definida a um estado representado pela combinação linear entre dois estados de helicidades diversas.
} 
[7] R.D. McKeown and P. Vogel, Phys. Rep. 395, 315 (2004).

[8] A series of papers on Super-Kamiokande data about atmospheric neutrino fluxes, Superkamiokande Collaboration,

S. Fukuda et al., Phys. Lett. B537, 179 (2002),

S. Fukuda et al., Phys. Rev. Lett. 86, 5651 (2001),

S. Fukuda et al., Phys. Rev. Lett. 85, 3999 (2000).

[9] A series of papers on SNO data about solar neutrino oscillation, SNO Collaboration,

Q.R. Ahmad et al., Phys. Rev. Lett. 89, 011302 (2002), Q.R. Ahmad et al., Phys. Rev. Lett. 89, 011301 (2002), Q.R. Ahmad et al., Phys. Rev. Lett. 87, 071301 (2001).

[10] K. Egushi et al., KamLAND Collaboration, First results from KamLAND: Evidence for reactor antineutrino disappearance, Phys. Rev. Lett. 90, 021802 (2003).

[11] C. Athanassopoulos et al., LSND Collaboration, Phys. Rev. Lett. 81, 1774 (1998).

[12] A. Aguilar et al., LSND Collaboration, Phys. Rev. D64, 112007 (2001).

[13] A. Bandyopadhyay, S. Choubey, S. Goswami, S.T. Petcov and D.P. Roy, Phys. Lett. B583, 134 (2004).

[14] L. Alvarez-Gaumé et al., PDG Collaboration, Review of Particle Physics in Neutrino Mass, Mixing and Flavor Change, edited by B. Kayser, Phys. Lett. B592, 145 (2004).

[15] B. Armbruster et al., KARMEN Collaboration, Phys. Rev. D65, 112001 (2002).

[16] A. Aguilar et al., MiniBoonE Collaboration, hepex/0408074.

[17] M. Gell-Mann, P. Ramond and R. Slansky, Complex Spinors And Unified Theories in Supergravity (North Holland, Amsterdan, 1979).

[18] T. Yanagida, in Proc. of the Workshop on Unified Theory and the Baryon Number of the Universe (KEK, Japan, 1979).

[19] J.C. D'Olivo, J.F. Nieves and P.B. Pal, Phys. Rev. Lett. 64, 1088 (1990).
[20] G. Barenboim, J. Bernabéu and O. Vives, Phys. Rev. Lett. 77, 3299 (1996)

[21] J.C. D'Olivo and J.F. Nieves, Phys. Lett. B383, 87 (1996)

[22] A.E. Bernardini and S. De Leo, a ser publicado em Mod. Phys. Lett. A20, 681 (2005)

[23] C. Itzykinson and J.B. Zuber, Quantum Field Theory (Mc Graw-Hill Inc., New York, 1980).

[24] J.J. Sakurai, Advanced Quantum Mechanics (AddisonWesley Publishing Company, New York, 1987).

[25] A.E. Bernardini and S. De Leo, Eur. Phys. J. C37, 471 (2004).

[26] S. De Leo and P. Rotelli, Int. J. Theor. Phys. 37, 2193 (1998).

[27] R.N. Mohapatra and J.C. Pati, Phys. Rev. D11, 566 (1975).

[28] S. Weinberg, The Quantum Theory of Fields (Cambridge University Press, New York, 1995).

[29] M.E. Peskin and D.V. Schroeder, An Introduction to Quantum Field Theory (Addison-Wesley Publishing Company, New York, 1995).

[30] F. Pisano and V. Pleitez, Phys. Rev. D46, 410 (1992).

[31] V. Pleitez and M.D. Tonasse, Phys. Rev. D48, 2353 (1993).

[32] F. Pisano and V. Pleitez, Phys. Rev. D51, 3855 (1995).

[33] H.N. Long and P.B. Pal, Mod. Phys. Lett. A13, 2355 (1998).

[34] S.L. Glashow, Nucl. Phys. 20, 579 (1961), A. Salam, Elementary Particle Theory (N. Svartholm, Stocholm, 1968), p. 367.

[35] S. Weinberg, Phys. Rev. Lett. 19, 1264 (1967).

[36] H. Georgi and S.L. Glashow, Phys. Rev. Lett. 32, 438 (1974).

[37] A.E. Bernardini and S. De Leo, a ser publicado em Phys. Rev. D, 076008-1 (2005). 